\title{
Cystic Neoplasm of the Pancreas
}

\author{
Alessandra Pulvirenti ${ }^{1} \cdot$ Giovanni Marchegiani $^{1} \cdot$ Giuseppe Malleo $^{1} \cdot$ Alex Borin $^{1}$. \\ Valentina Allegrini ${ }^{1} \cdot$ Claudio Bassi $^{1} \cdot$ Roberto Salvia $^{1}$
}

Received: 24 September 2015 / Accepted: 30 September 2015 /Published online: 28 October 2015

(C) Association of Surgeons of India 2015

\begin{abstract}
Cystic neoplasms of the pancreas (CNPs) are not considered as rare entities any more. Imaging-based population studies attested an overall prevalence of $2 \%$, but that becomes five times higher on individuals of more than 70 year old. This family of neoplasms includes a wide spectrum of benign, borderline, and malignant lesions whose actual biological behavior has not been completely clarified yet. Moreover, the management of CNPs still represents a challenge for gastrointestinal (GI) specialists. While many CNPs have an indolent behavior that justifies surveillance, others should be resected because of the risk of progression to invasive cancer. Due to the high morbidity related to pancreatic resections, the surgeon should balance very carefully the advantages of a radical resection with the risks of an unrequested dangerous procedure. We reviewed the current issues regarding CNPs, with a particular focus on the clinical and radiological features that are integrated in the current guidelines and that drive the management of these patients.
\end{abstract}

Keywords Cyst neoplasm $\cdot$ IPMN $\cdot$ Pancreatic cancer

\section{Introduction}

Until the last decade, cystic neoplasms of the pancreas (CNPs) were considered to be a rare entity. Nowadays, these

Claudio Bassi

claudio.bassi@univr.it

1 Unit of General and Pancreatic Surgery, The Pancreas Institute, G.B. Rossi Hospital, University of Verona Hospital Trust, P. Le L.A. Scuro 10, Verona 37134, Italy neoplasms are increasingly diagnosed, mainly because of the widespread use of cross-sectional imaging, particularly in asymptomatic patients undergoing radiological studies for non-pancreatic indications. The prevalence of CNPs is approximately $2.6 \%$ [1] and includes a broad spectrum of benign, borderline, and malignant lesions (Appendix). The biological behavior or these lesions is not completely clarified yet. In particular, the debate on the natural history of mucinous neoplasm is still open. This specific cohort encompasses a wide spectrum of neoplasms, all potentially precursors to invasive ductal adenocarcinoma. On the other hand, the indication to resect all these tumors is limited by the morbidity for pancreatic resections. In high-volume centers, morbidity for pancreatic resection has decreased in the last decades; however, it still remains as high as $30 \%-50 \%$ [2] and includes long-term sequelae. This evidence has generated controversies about the management of patients diagnosed with cystic lesions of the pancreas, whether a surgical operation should be offered or, alternatively, patients should be enrolled in surveillance protocols with periodic imaging would be the option of choice.

\section{Serous Cystic Neoplasm}

Serous cystic neoplasms (SCNs) represent about 10-16\% [3] of all resected cystic tumors of the pancreas. These neoplasms are composed of non-atypical cuboidal, glycogen-rich, epithelial cells that produce a watery fluid. Typically, they do not produce mucin and do not communicate with the pancreatic ductal system. Five macroscopic variants of $\mathrm{SCN}$ have been described: microcystic, macrocystic or oligocystic, mixed micro-macrocystic, Von Hippel-Lindau-associated, and solid. The majority of these tumors are benign (serous cystadenomas), with only few case reports of malignant 
variants (serous cystadenocarcinomas). The mean age of patients diagnosed with a SCN ranges from 52 years [4] to 62 years [3], depending from the series. Women are more frequently affected with an 8:1 ratio [4]. SCNs can occur in every aspect of the pancreatic gland; however, there seems to be a trend towards a more frequent origin in the head [4]. SCNs are usually asymptomatic and thus incidentally discovered, typically at cross-sectional imaging performed for unrelated complaints. When present, the most common symptoms are not specific, such as abdominal discomfort and mild epigastric pain (47-75\%) [3, 4]. Other symptoms are less frequent and could depend on the lesion location, like jaundice (4\%) [4], and from the lesion size, like palpable mass or obstruction of the upper gastrointestinal tract $(6.8 \%)[3,4]$.

$\mathrm{SCN}$ can be diagnosed by cross-sectional imaging in the vast majority of cases but limitations exist. Magnetic resonance imaging (MRI) plays the major role in the workup of these patients, due to its ability to characterize in details both the pancreatic parenchyma and the ductal system (Fig. 1). Due to this reason, it helps to distinguish microcystic SCNs from intraductal tumors of the peripheral branches (BD-IPMNs). The computed tomography (CT) appearance of a SCN depends on the macroscopic features of the tumor. Microcystic serous cystadenoma appears like a "honeycomb" with a typical central calcification named "central scar." However, less common macrocystic variants may be more difficult to differentiate from a mucinous tumor, with several studies having shown that CT is discriminatory in less than half of the cases $[5,6]$. When tomographic imaging is not able to provide a proper specific characterization, endoscopic ultrasound (EUS) and cyst fluid aspiration have been recommended as useful diagnostic techniques. EUS is particularly helpful in defining the morphology of the cystic lesion and excluding its connection with the ductal system, while the intra-cystic CEA level $(<200 \mathrm{ng} / \mathrm{ml})$ is highly diagnostic in excluding a mucinous neoplasm [7].

Surgical resection should be offered to patients who are symptomatic and surgically fit as well as every time that the tumor cannot be distinguished from other cystic neoplasms with uncertain biologic behavior. Particular

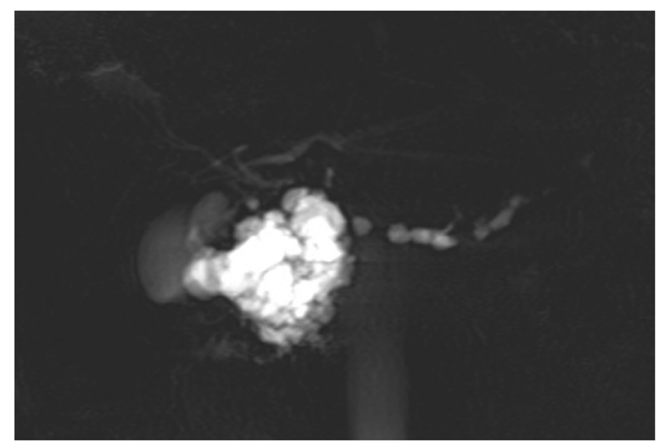

Fig. 1 Serous cystic neoplasm, head of the pancreas attention should be paid to patients with oligocystic/ macrocystic variant, with a medical history of nonpancreatic malignancies, and for elderly patients because of the faster growth pattern that has been shown in these cases [8]. Once a surgical indication exists, minimally invasive technique should be taken into account, even though SCNs requiring surgery are of giant dimensions in several cases. Tumor size has been historically considered as a criterion for resection, even without the presence of mass-related symptoms; however, this represents now a matter of debate. In a recent series, Tseng et al. [9] suggested a diameter cutoff of $4 \mathrm{~cm}$ for operative decision. Conversely, a study from our institution [8] suggested that tumor size at the first observation is not related with the growth pattern and should not be used for decisional purposes. A surveillance protocol with MRI and magnetic resonance cholangio-pancreaticography (MRCP) can be proposed for all patients with well-characterized and asymptomatic SCN. A follow-up time frame of 2 years seems to be appropriate.

\section{Mucinous Cystic Neoplasm}

Mucinous cystic neoplasm (MCN) is the second most frequent cystic neoplasm of the pancreas, representing $23 \%$ [2] of all resected pancreatic cysts. MCNs occur almost exclusively in female, such as male cases are reported to be extremely rare [9-10]. The median age of presentation is reported to be 48 (range 16-82) and tumors are preferentially located in the body/tail of the pancreas [11, 12]. Patients with malignant MCNs are typically older, suggesting a time-related degeneration of the tumor from an initially benign lesion. From the pathological standpoint, MCNs are defined by the presence of mucin-producing neoplastic epithelial cells lining the cyst, and a characteristic "ovarian-type" subepithelial stroma [13]. The epithelial layer can exhibit various degrees of atypia, ranging from adenoma to invasive carcinoma, that frequently coexist in the same lesion. A communication between the cyst and the pancreatic ductal system is not present and this feature is diagnostic in order to differentiate MCNs from intraductal papillary mucinous neoplasms (IPMNs). The clinical presentations of MCNs can be extremely various. Almost half of the patients are asymptomatic and diagnosis is made incidentally. However, when present, the most frequent symptoms are not specific: namely, abdominal discomfort, nausea, and pain [14]. Weight loss and anorexia are considered to be worrisome symptoms, correlating with a higher likelihood of malignancy of the lesion.

Typically, the radiological aspect of MCN is a thick-walled single cyst located in the body or tail of the pancreas, often 


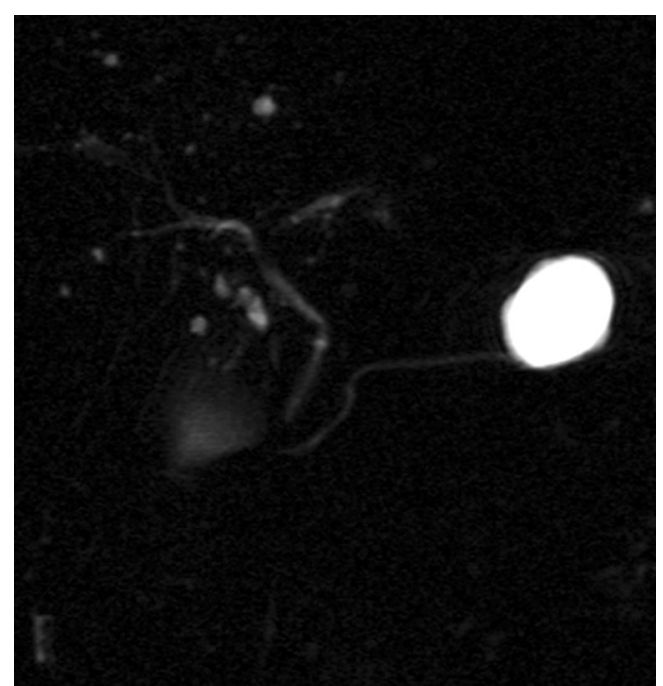

Fig. 2 Mucinous cystic neoplasm, tail of pancreas

associated with thin septae delimiting the cystic spaces and calcifications (Fig. 2). The differential diagnosis of small cysts located in the distal pancreas might be difficult. The most challenging one is between MCNs and macrocystic/ oligocystic SCNs, and often the surgical indication is driven by this diagnostic uncertainty. The gold standard to assess the features of MCNs is the CT scan, as the MRI with MRCP fails to detect the calcifications, which could be present and correlate with malignancy [15]. However, MRCP is capable to detect the lack of a communication between the cyst and the ductal system with a higher accuracy, thus an IPMN can be excluded. If a clinical history of pancreatitis is present, a diagnosis of pseudocyst should be excluded, and in this scenario EUS can be beneficial $[16,17]$. Large surgical series have shown that the risk of invasive cancer for MCNs is 3.9$11 \%$ and the 5 -year disease-specific survival is $100 \%$ and $58-62.5 \%$ for non-invasive and invasive tumors, respectively [11]. In the last decades, when a MCN was suspected, indication to surgery was given by young patients' age because of the risk of progression to pancreatic cancer and by the relatively low morbidity associated to the distal resection of the pancreas [18]. Recently, several studies have shown that a conservative approach could be feasible in selected cases such as small MCNs without solid component in patients with high risk for surgery $[12,14]$.

\section{Intraductal Papillary Mucinous Neoplasm}

IPMNs have represented the "new kid on the block" of pancreatic cysts. This family of neoplasms was described for the first time by Ohashi [19] in 1982, and until the late 1990s was considered extremely rare. In 1996, IPMN was included in the World Health Organization (WHO) classification (Table 1) [21]. We are
Table 1 Pancreas cystic tumors WHO classification 2010 [20]

\begin{tabular}{l} 
Histological Classification of Pancreatic Cystic Tumours \\
\hline Benign \\
Acinar cell cystadenoma \\
Serous cystadenoma \\
Premalignant lesions \\
Intraductal papillary mucinous neoplasms with low- or intermediate- \\
grade dysplasia \\
Intraductal papillary mucinous neoplasms with high-grade dysplasia \\
Intraductal tubulopapillary neoplasms \\
Mucinous cystic neoplasms with low- or intermediate-grade dysplasia \\
Mucinous cystic neoplasms with high-grade dysplasia \\
Malignant \\
Acinar cell cystoadenocarcinoma \\
Intraductal papillary mucinous neoplasms with an associated invasive \\
carcinoma \\
Mucinous cystic neoplasms with an associated invasive carcinoma \\
Serous cystoadenocarcinoma \\
Solid-pseudopapillary neoplasms
\end{tabular}

nowadays aware that IPMNs are the most frequently detected pancreatic cysts, representing $39 \%$ of the cystic surgical series [8]. Patients undergoing resection are typically in their sixth decade of life [11] with a slightly higher incidence for males. IPMNs can be either sporadic or associated with familial syndromes such as Peutz-Jeghers syndrome (PJS) and familial adenomatous polyposis (FAP). Furthermore, some authors reported a higher incidence of extrapancreatic neoplasm among patients [22] with IPMN. However, a recent study from Verona does not confirm these data [23].

More than $80 \%$ of IPMNs are detected incidentally in asymptomatic patients, while, when present, symptoms include abdominal pain, weight loss, jaundice, and acute pancreatitis [23]. In two thirds of cases, IPMNs are located in the proximal pancreas, and in $8 \%$ they could be multifocal affecting the entire gland. From the pathological standpoint, IPMNs are defined as "massforming pre-invasive neoplasms growing within the ducts of the pancreas" [24]. The epithelium proliferation, associated with papillary growth, causes an overproduction of mucus resulting in a gross dilatation of pancreatic ducts. In terms of dysplasia, this can be defined into low-, intermediate-, or high- grade. Hence, an invasive component can be present. Neoplastic proliferation can occur in either just the main duct (MD-IPMN) or the side branches of the pancreatic ductal system (BD-IPMN) or a combination of side branches and the main duct (mixed or combined IPMN). Furthermore, different epithelial subtypes have been described according to the histology and mucin immunophenotype, namely, the gastric, intestinal, pancreatobiliary, and oncocytic. These subtypes have been showed to be predictors of disease biology and postoperative outcomes. 


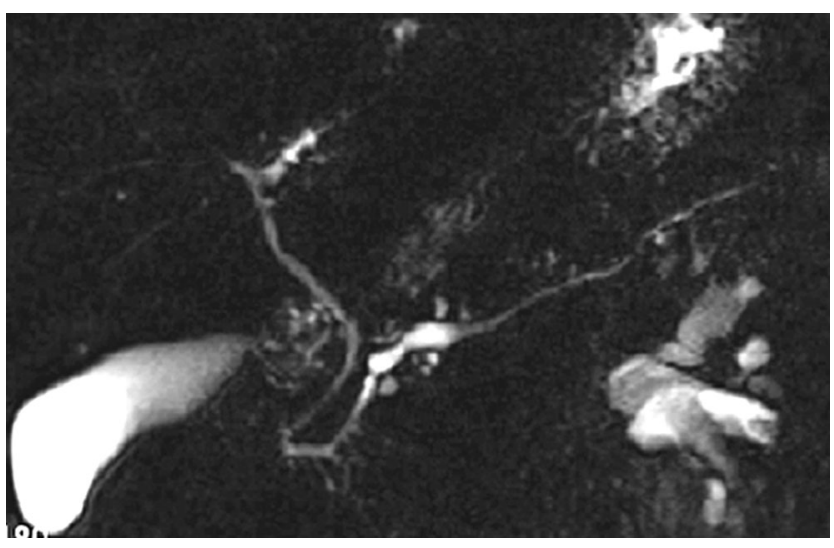

Fig. 3 Segmental intraductal papillary mucinous neoplasm of the main duct, head of pancreas

The International Association of Pancreatology published recommendations (Sendai guidelines) for the management of IPMN in 2006 and subsequent revision (Fukuoka guidelines) in 2012 $[18,25]$. According to the guidelines, resection is warranted for MD-IPMN, since these lesions have a high prevalence of high-grade dysplasia/invasive carcinoma and a 5-year survival rate of $31-54 \%$. Once the main pancreatic duct (MPD) is less than $10 \mathrm{~mm}$ in diameter, and neither symptoms nor mural nodules are present, a strict follow-up protocol can be suggested. In partial contrast with these recommendations, a recent study suggested that primary surveillance seems to be feasible in selected patients affected by mixed-type IPMN. In particular, the progression to an invasive carcinoma was described in only $13 \%$ of cases after $4-5$ years of follow-up [ 26 , 27]. This evidence pictures how, even if extremely valuable in the clinical practice, the guidelines are not comprehensive and need further validation and modification through large, prospective, cohort studies.

As for BD-IPMNs, the Fukuoka guidelines recommend resection when the so called "high-risk stigmata" (obstructive jaundice, presence of enhancing solid component within cyst, MPD size of $\geq 10 \mathrm{~mm}$ ) occur.

As suggested by the guidelines, the first goal in the radiological assessment of IPMNs is to diagnose the main pancreatic duct involvement and the mural nodules presence. In this regard, both CT scan and MRI with MRCP have demonstrated high accuracy (Figs. 3 and 4); however, magnetic resonance is more effective in depicting the communication between the IPMN and the ductal system [28, 29]. EUS can be used as a second level examination

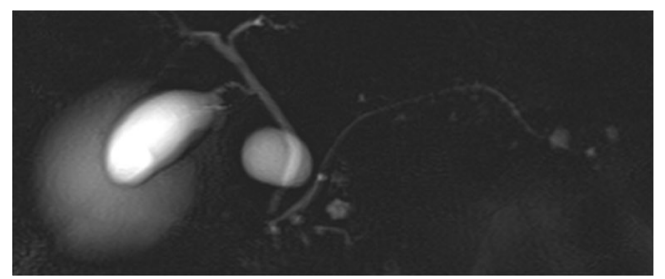

Fig. 4 Multifocal intraductal papillary mucinous neoplasm of the branch ducts

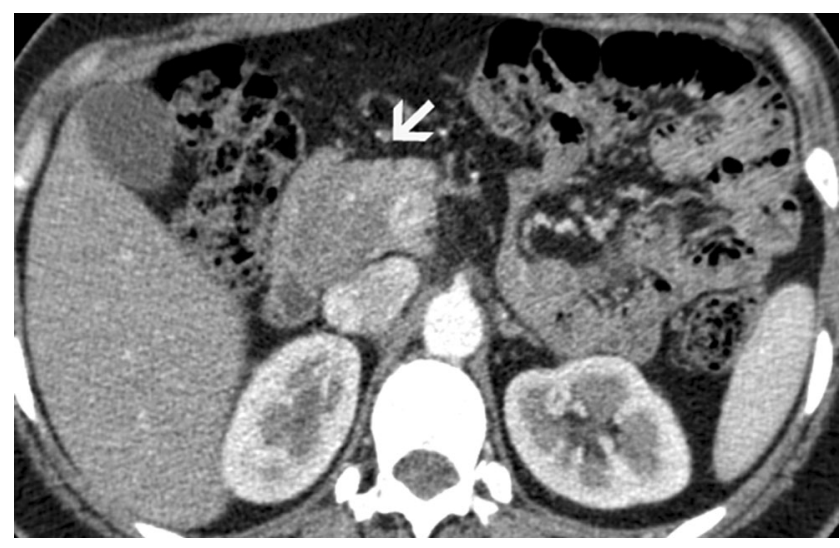

Fig. 5 White arrow Solid pseudopapillary neoplasm, head of the pancreas

in patients with "worrisome features" (cyst $>3 \mathrm{~cm}$, thickened enhanced cyst walls, MPD size of 5-9 mm, non-enhanced mural nodules, abrupt change in the MPD caliber, and lymphadenopathy) for detecting mural nodules and it allows fluid aspiration and targeted biopsies. Analyses of the CEA and amylase levels as well as cytology of the cyst content are often useful, but fail to discriminate MCN from IPMN.

\section{Solid Pseudopapillary Neoplasm}

Solid pseudopapillary neoplasms (SPNs) are so far the less common resected pancreatic cystic tumors, accounting for about $3 \%$ [2] of total. The most common affected individuals are female in young age, between 30 and 40 years old [30-32]. SPN can be located in any portion of the pancreas and vague abdominal pain is the most common symptom; thus, it is usually related to cyst volume. In the vast majority of cases, SPN is a slowly growing, indolent tumor with a low grade of malignancy. However, metastatic presentation could occur at the time of diagnosis in up to $10-15 \%$ of patients [33]. CT scan allows the diagnosis by demonstrating a vascularized and encapsulated mass with definite margins. Calcifications and septa may be present, but the pathognomonic sign is represented by the alternation of solid and cystic areas (Fig. 5). A common diagnostic misinterpretation can occur with cystic neuroendocrine tumors, mainly because of the rich vascularization. A positron emission tomography (PET)-CT with $\mathrm{Ga}^{68}$ could be helpful for the differential diagnosis. Most recent series showed how the prognosis after surgical resection is favorable, even for SPNs with systemic involvement $[32-35,36]$. For this reason, surgery must be considered in all patients, including in the presence of metastases, since longterm survival can be still achieved [36].

\section{Compliance with Ethical Standards}

Conflict of Interest The authors declare that they have no competing interests. 


\section{Appendix}

Table 2 Clinical features and diagnostic imaging

\begin{tabular}{|c|c|c|}
\hline Cyst type & Clinical features & Imaging \\
\hline $\begin{array}{c}\text { Serous cystic } \\
\text { neoplasm }\end{array}$ & $\begin{array}{l}\text { - Female/male 8:1 } \\
\text { - Asymptomatic } \\
\text { - Benign }\end{array}$ & $\begin{array}{l}\text { - Head/neck/body/tail } \\
\text { - Honeycomb aspect } \\
\text { - Central scar } \\
\text { - No communication } \\
\text { with the ductal system } \\
\text { - Macrocystic/oligocystic } \\
\text { variant could not be } \\
\text { differentiated from } \\
\text { MCN }\end{array}$ \\
\hline $\begin{array}{l}\text { Mucinous cystic } \\
\text { neoplasm }\end{array}$ & $\begin{array}{l}\text { - Woman } \\
\text { - Asymptomatic or } \\
\text { abdominal } \\
\text { discomfort } \\
\text { - Premalignant and } \\
\text { malignant lesion }\end{array}$ & $\begin{array}{l}\text { - Body/tail } \\
\text { - Thick-walled single } \\
\text { cysts } \\
\text { - No communication } \\
\text { with the ductal system }\end{array}$ \\
\hline $\begin{array}{l}\text { Intraductal papillary } \\
\text { mucinous } \\
\text { neoplasm }\end{array}$ & $\begin{array}{l}\text { - Equal gender } \\
\text { distribution } \\
\text { - Mostly } \\
\text { Asymptomatic } \\
\text { - Main duct variant: } \\
\text { high incidence of } \\
\text { malignancy } \\
\text { - Branch duct variant: } \\
\text { low risk of } \\
\text { malignancy }\end{array}$ & $\begin{array}{l}\text { MD-IPMN } \\
\text { - } 2: 3 \text { head/neck } \\
\text { - Dilatation of the main } \\
\text { pancreatic duct } \\
\text { BD-IPMN } \\
\text { - Mostly at the head but } \\
\text { body tail location is } \\
\text { possible } \\
\text { - Cyst or cluster of cyst } \\
\text { without dilatation of } \\
\text { the main pancreatic } \\
\text { duct } \\
\text { - Communication with } \\
\text { the ductal system }\end{array}$ \\
\hline $\begin{array}{l}\text { Solid pseudopapillary } \\
\text { neoplasm }\end{array}$ & $\begin{array}{l}\text { - Young woman } \\
\text { - Asymptomatic } \\
\text { or abdominal } \\
\text { discomfort } \\
\text { - Malignant lesion }\end{array}$ & $\begin{array}{l}\text { - Head/neck/body/tail } \\
\text { - Well vascularized } \\
\text { and encapsulated } \\
\text { - Alternation of solid } \\
\text { and cystic area } \\
\text { - No communication } \\
\text { with the ductal system } \\
\text { - PET-CT with Ga68 } \\
\text { to differentiate from } \\
\text { cystic neuroendocrine } \\
\text { tumors }\end{array}$ \\
\hline
\end{tabular}

\section{References}

1. Laffan TA, Horton KM, Klein AP et al (2008) Prevalence of unsuspected pancreatic cysts on MDCT. AJR Am J Roentgenol 191(3):802-7

2. Valsangkar NP, Morales-Oyarvide V, Thayer SP et al (2012) 851 resected cystic tumors of the pancreas: a 33-year experience at the Massachusetts General Hospital. Surgery 152(3 Suppl 1):S4-12

3. Galanis C, Zamani A, Cameron JL et al (2007) Resected serous cystic neoplasms of the pancreas: a review of 158 patients with recommendations for treatment. J Gastrointest Surg 11(7):820-6

4. Bassi C, Salvia R, Molinari E, Biasutti C, Falconi M, Pederzoli P (2003) Management of 100 consecutive cases of pancreatic serous cystadenoma: wait for symptoms and see at imaging or vice versa? World J Surg 27(3):319-23

5. Curry CA, Eng J, Horton KM et al (2000) CT of primary cystic pancreatic neoplasms: can CT be used for patient triage and treatment? AJR Am J Roentgenol 175:99-103

6. Procacci C, Biasiutti C, Carbognin G et al (1999) Characterization of cystic tumors of the pancreas: CT accuracy. J Comput Assist Tomogr 23:906-912

7. Brugge WR, Lewandrowski K, Lee-Lewandrowski E et al (2004) Diagnosis of pancreatic cystic neoplasms: a report of the cooperative pancreatic cyst study. Gastroenterology 126(5):1330-1336

8. Malleo G, Bassi C, Rossini R et al (2012) Growth pattern of serous cystic neoplasms of the pancreas: observational study with longterm magnetic resonance surveillance and recommendations for treatment. Gut 61(5):746-51

9. Tseng JF, Warshaw AL, Sahani DV et al (2005) Serous cystadenoma of the pancreas: tumor growth rates and recommendations for treatment. Ann Surg 242(3):413-419

10. Regi P, Salvia R, Cena C, Girelli R, Frigerio I, Bassi C (2013) Cystic "feminine" pancreatic neoplasms in men. Do any clinical alterations correlate with these uncommon entities? Int J Surg 11(2):157-60

11. Crippa S, Fernández-Del Castillo C, Salvia R et al (2010) Mucinproducing neoplasms of the pancreas: an analysis of distinguishing clinical and epidemiologic characteristics. Clin Gastroenterol Hepatol 8(2):213-9

12. Yamao K, Yanagisawa A, Takahashi PK (2011). Clinicopathological features and prognosis of mucinous cystic neoplasm with ovarian-type stroma.; 40(1):67-71

13. Zamboni G, Fukushima N, Hruban RH, Klöppel G (2010) Mucinous cystic neoplasms of the pancreas. In: Bosman FT, Carneiro F, Hruban RH, Theise ND (eds) WHO classification of tumours of the digestive system, 4th edn. IARC, Lyon, pp 300-303

14. Nguyen D, Dawson DW, Hines OJ, Reber HA, Donahue TR (2014) Mucinous cystic neoplasms of the pancreas : are we overestimating malignant potential ? Am Surg 80(10):915-9

15. Scott J, Martin I, Redhead D, Hammond P, Garden OJ (2000) Mucinous cystic neoplasms of the pancreas: imaging features and diagnostic difficulties. Clin Radiol 55(3):187-92

16. Manfredi R, Ventriglia A, Mantovani W et al (2015) Mucinous cystic neoplasms and serous cystadenomas arising in the body-tail of the pancreas: MR imaging characterization. Eur Radiol 25(4): 940-9

17. Sahani DV, Kadavigere R, Saokar A, Fernandez-del Castillo C, Brugge WR, Hahn PF (2005) Cystic pancreatic lesions: a simple imaging-based classification system for guiding management. Radiographics 25(6):1471-84

18. Tanaka M, Fernández-del Castillo C, Adsay V et al (2012) International consensus guidelines 2012 for the management of IPMN and MCN of the pancreas. Pancreatology 12(3):183-97

19. Ohashi K, Murakami Y, Maruyama M (1982) Four cases of mucinproducing cancer of the pancreas on specific findings of the papilla of Vater [in Japanese]. Prog Dig Endoscopy 20:348-351

20. Bosman FT, Carneiro F, Hruban RH, Theise ND (2010) WHO classification of tumours of the digestive system, 4th edn. International Agency for Research on Cancer, Lyon

21. Kloppel G, Solcia E, Longnecker DS et al (1996) World Health Organization international histological typing of tumors of the exocrine pancreas. Springer, Berlin, pp 1-61

22. Kawakubo K, Tada M, Isayama $H$ et al (2011) Incidence of extrapancreatic malignancies in patients with intraductal papillary mucinous neoplasm of the pancreas. Gut 60:12491253

23. Marchegiani G, Mino-Kenudson M, Sahora K, et al. (2014). IPMN involving the main pancreatic duct: biology, epidemiology, and long-term outcomes following resection. Ann Surg 261(5):976-83 
24. Adsay V, Mino-Kenudson M, Furukawa T, et al. (2015). Pathologic evaluation and reporting of intraductal papillary mucinous neoplasms of the pancreas and other tumoral intraepithelial neoplasms of pancreatobiliary tract: recommendations of Verona Consensus Meeting. Ann Surg, Mar 13

25. Tanaka M, Chari S, Adsay V, Fernández-del Castillo C, Falconi M, Shimizu M, International Association of Pancreatology et al (2006) International consensus guidelines for management of intraductal papillary mucinous neoplasms and mucinous cystic neoplasms of the pancreas. Pancreatology 6(1-2):17-32

26. Roch AM, Ceppa EP, Al-Haddad MA et al (2014) The natural history of main duct-involved, mixed-type intraductal papillary mucinous neoplasm: parameters predictive of progression. Ann Surg 260(4):680-8, discussion 688-90

27. Sahora K, Fernández-del Castillo C, Dong F et al (2014) Not all mixed-type intraductal papillary mucinous neoplasms behave like main-duct lesions: implications of minimal involvement of the main pancreatic duct. Surgery 156(3):611-21

28. Ogawa H, Ito S, Ikeda $\mathrm{M}$ et al (2008) Intraductal papillary mucinous neoplasm of the pancreas: assessment of the likelihood of invasiveness with multisection CT. Radiology 248:876-886

29. Sahani DV, Kadavigere R, Blake M et al (2006) Intraductal papillary mucinous neoplasm of the pancreas: multi-detector row CT with 2D curved reformations: correlation with $\mathrm{CT}$. Radiology 238:560-569
30. Reddy S, Cameron JL, Scudiere J et al (2009) Surgical management of solid-pseudopapillary neoplasms of the pancreas (Franz or Hamoudi tumors): a single-institutional series. J Am Coll Surg 208:950-959

31. Papavramidis T, Papavramidis S (2005) Solid pseudopapillary tumors of the pancreas: review of 718 patients reported in English literature. J Am Coll Surg 200(6):965-72

32. Butte JM, Brennan MF, Gonen M et al (2011) Solid pseudopapillary tumors of the pancreas. Clinical features, surgical outcomes, and long-term survival in 45 consecutive patients from a single center. J Gastrointest Surg 15:350-357

33. Martin RC, Klimstra DS, Brennan MF at al (2002) Solidpseudopapilary tumor of the pancreas: a surgical enigma? Ann Surg Oncolo 9:35-40

34. Yu P, Cheng X, Du Y, et al. (2015). Solid pseudopapillary neoplasms of the pancreas: a 19-year multicenter experience in China. J Gastrointest Surg. May 23

35. Kang CM, Choi SH, Kim SC, Lee WJ, Choi DW, Kim SW (2014) Predicting recurrence of pancreatic solid pseudopapillary tumors after surgical resection: a multicenter analysis in Korea. Ann Surg 260(2):348-55

36. Estrella JS, Li L, Rashid A et al (2014) Solid pseudopapillary neoplasm of the pancreas: clinicopathologic and survival analyses of 64 cases from a single institution. Am J Surg Pathol 38(2):147-57 